\title{
Ruptured ectopic pregnancy in the rudimentary horn of bicornuate uterus: a case report
}

\section{Hema S. Patil*, Neha Gupta}

Department of Obstetrics \& Gynecology, JNMC, Belgaum, Karnataka, India

Received: 9 December 2013

Accepted: 15 December 2013

\author{
*Correspondence: \\ Dr. Hema S. Patil, \\ E-mail: hemabanad@yahoo.com
}

(c) 2014 Patil HS et al. This is an open-access article distributed under the terms of the Creative Commons Attribution Non-Commercial License, which permits unrestricted non-commercial use, distribution, and reproduction in any medium, provided the original work is properly cited.

\begin{abstract}
Rupture uterus in first and second trimester in a non-scarred uterus is generally seen with associated mullerian anomalies. A 22 year old primigravida with 16 weeks gestational age presented with pain in abdomen since 6 hours prior to admission and hypovolemic shock. Though she had taken regular antenatal visits and a transabdominal scan at 14-16wks with the report opining a normal study, the diagnosis of the fetus in the rudimentary horn was missed. A provisional diagnosis of rupture of right rudimentary horn of a bicornuate uterus was made afet clinical examination and the TVS showing the cervix continuing into a normal uterus with no fetus within it and a vague right adnexal mass. Transabdominal scan also showed vague hypoechoic mass in the right adnexa with a fetus of 16-17 weeks above the mass with absent cardiac activity. Emergency laparotomy with resuscitation and excision of the ruptured horn and blood transfusion saved the life of the patient.
\end{abstract}

Keywords: Bicornuate uterus, Rudimentary horn, Ruptured ectopic

\section{INTRODUCTION}

Mullerian duct anomalies result from defective fusion, canalization or absorption of the median septum of the female reproductive system during embryonic development. ${ }^{1}$ A working classification depending on the degree of failure of normal development was proposed by Buttram and Gibbons in 1979 and modified in 1988 by American Society of Reproductive Medicine. ${ }^{1}$ The prevalence of uterine malformation is estimated to be $6.7 \%$ in the general population, slightly higher $(7.3 \%)$ in the infertility population, and significantly higher in a population of women with a history of recurrent miscarriages $(16 \%))^{2}$ Presence of bicornuate uterus predisposes to lower fertility, high risk of abortions, preterm births. IUGR, malpresentations and lower fetal survival rate. However after corrective surgery successful pregnancy with good outcome is achieved in $80 \%$ of cases. ${ }^{1}$ Ruptured horn poses a life threatening situation to the pregnant woman. We report a case of 22 year old primigravida with ruptured right rudimentary horn of a bicornuate uterus.

\section{CASE REPORT}

A 22 year- old primigravida with 16 weeks gestation according to LMP presented with severe abdominal pain since 6 hours prior to admission. On examination there was tachycardia, feeble pulse, a blood pressure of 90/60 $\mathrm{mmHg}$ with cold extremities and pallor suggestive of hypovolemic shock. Abdomen appeared distended with guarding and rigidity present. Bowel sounds were absent. No uterine contour was appreciable. On per speculum examination a normal healthy cervix and vagina were observed. No bleeding. Per vaginal examination demonstrated cervical motion tenderness and uterine size and contour could not be appreciated.

Trans-vaginal ultrasound imaging showed normal cervical canal, normal sized uterus, hemoperitoneum and a vague hypoechoic mass in pouch of Douglas and right 
adnexa suggestive of placental mass. No intrauterine gestation was noted. Trans-abdominal scan showed a fetus of 16-17 weeks seen above the vague hypoechoic mass with no cardiac activity. Diagnosis of ruptured ectopic was made and patient was taken up for emergency laparotomy with resuscitation with blood and blood products. Intraoperative findings were hemoperitoneum of $2000 \mathrm{ml}$; ruptured rudimentary horn on right side with partially attached placenta; fetus in the right paracolic gutter; bicornuate uterus; left tube and ovaries were normal; right side ovary normal, right tube and round ligament was attached to the ruptured horn; it was classified as non-communicating rudimentary horn.

Patient had taken 2 antenatal visits earlier with a USG done at 15 weeks gestation that reported as normal intrauterine pregnancy. Timely suspicion and diagnosis with emergency laparotomy with resuscitation saved the patient's life.

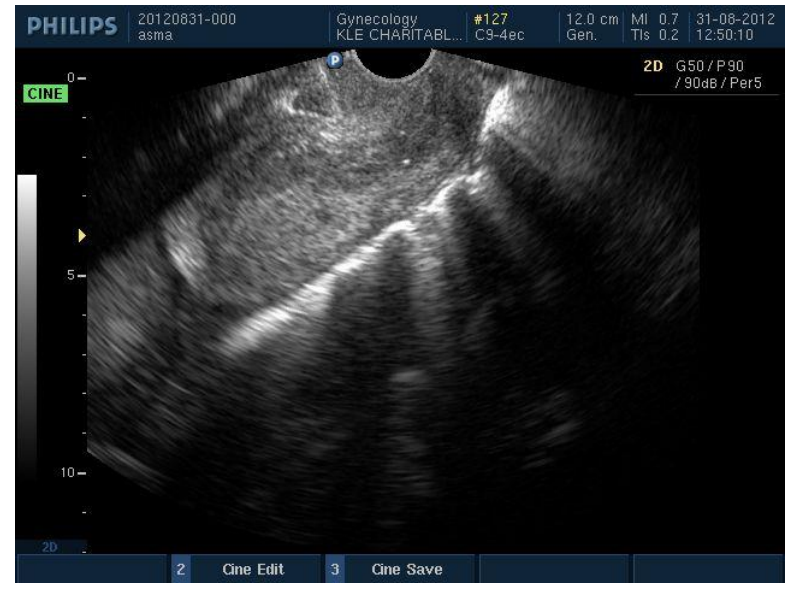

Figure 1: Trans-vaginal ultrasound imaging showed normal cervical canal, normal sized uterus, hemoperitoneum and a vague hypoechoic mass in pouch of Douglas and right adnexa suggestive of placental mass.

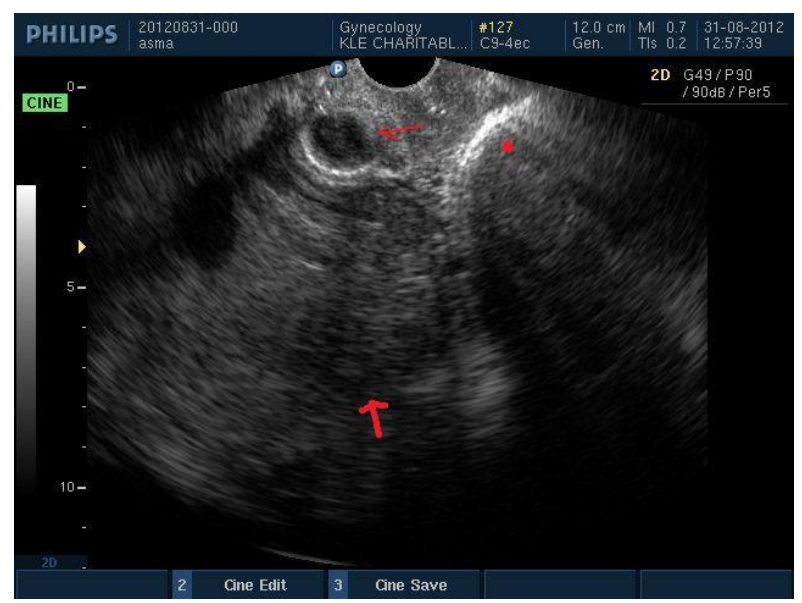

Figure 2: Trans-abdominal scan showed a fetus of 1617 weeks seen above the vague hypoechoic mass with no cardiac activity.

\section{DISCUSSION}

Bicornuate uterus results from non-fusion of the two cornua of the uterus. Estimated incidence of pregnancy in rudimentary horn is 1 in 10000 to 1 in $40000 .^{3}$ Most likely mechanism of a pregnancy in a noncommunicating rudimentary horn is transperitoneal migration of the spermatozoa or the fertilized ovum. ${ }^{4}$ Depending on the ability of the horn to undergo hypertrophy and its musculature the rupture occurs between 5 weeks to 35 weeks. $80 \%$ of rupture occurs before 20 weeks. The haemorrhage occurring because of rupture is massive and life threatening if corrective measures are not taken immediately. The usual outcome of rupture in second trimester in $90 \%$ of cases is fetal demise.

Primary strategy in ruptured horn is surgical removal and repair of the defect. ${ }^{5}$ Immediate surgery is recommended by most obstetricians even in a non-ruptured uterine horn pregnancy if diagnosed before catastrophe. ${ }^{6}$ A routine USG examination in suspected cases of uterine anomaly or an MRI is also appropriate to prevent the catastrophe. This patient had taken antenatal visits still the diagnosis was missed. What we like to emphasise is that simple and basic clinical skill like a per vaginal examination would've led to a suspicion of an adnexa mass, which could be confirmed and definitively diagnosed as rudimentary horn by TVS. Patient could've undergone a definitive elective surgery and the catastrophe avoided. Hence first trimester complete evaluation of the genital system and all other systems is a must in all pregnant patients to avoid and counsel regarding future catastrophe. High level of suspicion should be kept whenever a patient presents with acute abdomen with amenorrhoea.

\section{CONCLUSION}

With the advancements made in radio-diagnosis it is possible to detect the mullerian anomaly even in pregnancy. However the possibilities of missing them are higher in inexperienced hands. In absence of timely diagnosis or misdiagnosis the condition can be fatal. Timely diagnosis, emergency laparotomy, blood and blood products transfusion is the key to the management of a ruptured ectopic pregnancy.

\section{Funding: No funding sources \\ Conflict of interest: None declared \\ Ethical approval: Not required}

\section{REFERENCES}

1. Padubidri VG, Daftary SN. Disorders of the Ovary and Benign Tumours. In: Howkins and Bourne, eds. Textbook of Gynecology. $15^{\text {th }}$ ed. Haryana, India: Elsevier Print; 2013: 98-99.

2. Sotirios H. Saravelos, Karen A. Cocksedge and TinChiu Li. Prevalence and diagnosis of congenital 
uterine anomalies in women with reproductive failure: a critical appraisal. Human Reproduction Update. 2008; September-October;14(5):415-29.

3. Sfar E, Zine S, Bourghida S, Bettaieb A, Chelli H. Pregnancy in a rudimentary uterine horn: main clinical forms. 5 cases. Rev Fr Gynecol Obstet. 1994;89:21-6.

4. M. Scholtz. A full-time pregnancy in a rudimentary horn of the uterus. British Journal of Obstetrics \& Gynaecology. 1951;58:293-6.

5. G. Nahum. Rudimentary uterine horn pregnancy: the 20thcentury worldwide experience of 588 cases.
Journal of Reproductive Medicine. 2002;47(2):15163.

6. Y. Jayasinghe, A. Rane, H. Stalewski, S. Grover. The presentation and early diagnosis of the rudimentary uterine horn. Obstetrics and Gynecology. 2005;105(6):1456-67.

DOI: $10.5455 / 2320-1770$. ijrcog20140352

Cite this article as: Patil HS, Gupta N. Ruptured ectopic pregnancy in the rudimentary horn of bicornuate uterus: a case report. Int J Reprod Contracept Obstet Gynecol 2014;3:248-50. 